\title{
Laban Effort and Shape analysis of affective hand and arm movements
}

\author{
Ali-Akbar Samadani*, SarahJane Burton ${ }^{\dagger}$, Rob Gorbet ${ }^{\ddagger}$, and Dana Kulić* \\ ${ }^{*}$ Department of Electrical and Computer Engineering \\ University of Waterloo, Waterloo, Canada \\ Email: asamadan@uwaterloo.ca \\ ${ }^{\dagger}$ Department of Visual and Performing Arts, Sheridan College, Oakville, Canada \\ ${ }^{\ddagger}$ Centre for Knowledge Integration, University of Waterloo, Waterloo, Canada
}

\begin{abstract}
The Laban Effort and Shape components provide a systematic tool for a compact and informative description of the dynamic qualities of movements. To enable the application of Laban notation in computational movement analysis, measurable physical correlates of Effort and Shape components need to be identified. Such physical correlates enable quantification of Effort and Shape components, which in turn facilitates computational analysis of affective movements. In this work, two existing approaches to quantification of Laban Effort components (Weight, Time, Space, and Flow) based on measurable movement features (position, velocity, acceleration, and jerk) are adapted for hand and arm movements, and a new approach for quantifying Shape Directional based on the average trajectory curvature is proposed. The results show a high correlation between Laban annotations provided by a certified movement analyst (CMA) and the quantified Effort Weight $(81 \%)$, Time $(77 \%)$ and Shape Directional (93\%) for an affective hand and arm movement dataset.
\end{abstract}

\section{INTRODUCTION}

With the advancement of interactive technologies such as social robotics e.g., [1], kinetic sculptures e.g., [2], and animated agents e.g., [3], it is becoming more important to develop computational models that can accurately recognize users' expressions, and generate appropriate responses through different modalities including movement. The work on affect and its bodily manifestation in psychology and the dance community provides valuable insights that can advance the research in affective computing. In particular, movement notation systems provide a rich tool for a more compact and informative representation of the movements as compared to high-dimensional joint angle time-series trajectories. The existing notation systems can be divided into two classes: 1) functional, and 2) structural [4].

Functional approaches describe the communicative function of a displayed movement using verbal labels, i.e., they provide a taxonomy of the movements. The Ekman and Friesen formulation of kinesic ${ }^{1}$ behaviours into five categories (Emblems, Illustrators, Affect displays, Regulators, and $\mathrm{Ma}$ nipulators) is an example of a functional notation system [6]. The verbal labels used in functional notation systems provide no information about the appearance of the movements; hence, these systems are not adequate for detailed movement analysis and reproduction. Structural approaches notate the structure, dynamics, and form characteristics of the movements [4]. Inspired by linguistic notation systems, Birdwhistell proposed

\footnotetext{
${ }^{1}$ Birdwhistell defines non-verbal communicative body movements as $\mathrm{ki}$ nesics [5].
}

a structural movement notation system that parallels phonemic transcription and consists of kine (the smallest perceivable body motion e.g., raising eye brows), kinemes (a group of movements with the same social meaning e.g., one nod, two nod, three nods), kinemorphs (a combination of kinemes forming a gesture), followed by kinemorphic classes and complex kinemorphic constructs [5]. Birdwhistell also used motion qualifiers and action modifiers that define the extent of muscular tension, duration, and range of the movement.

The Laban system is a structural notation system that was developed for writing and analyzing dance choreography [7], [8]. Laban encodes both the structural characteristics and the expressive specificities of a movement using a set of semantic components. The Laban system has four major components: Body, Effort, Shape, and Space. Body and Space describe the structural characteristics of the movement, whereas Effort and Shape are primarily concerned with the qualitative aspects of the movement. Effort encodes the use of energy along four bipolar dimensions of Weight, Time, Space, and Flow with their extremes being Light/Strong, Sustained/Sudden, Indirect/Direct, and Free/Bound, respectively. Shape characterizes the dynamic changes in the movement using three components: Shape Flow, Directional, and Shaping/Carving [9].

Despite their proven suitability for movement coding, the notation systems do not explicitly provide quantitative measures, which is perhaps the main barrier to their application in affective computing. Furthermore, the extensive attention to microanalysis (e.g., Birdwhistell system [5]), and the need for special training (e.g., Laban system) hamper their adoption in affective computing.

In computational human motion analysis, movements are commonly represented in terms of joint angle trajectories (e.g., [10]), or derived discrete or time-series features (e.g., maximum velocity [11]). Movement modeling based on these kinematic features does not necessarily capture expressive qualities. Therefore, there is a need for a movement representation that can highlight the expressive qualities of the movements.

Laban Effort and Shape provide a consistent representation of the expressive qualities in terms of a fixed number of semantic components. There have been a few attempts to quantify Laban components. Nakata et al. proposed a quantification of the Effort components (Time, Weight, Space), and Shape components (Shaping/Carving and Shape Flow) for a pet robot [12]. The quantified components were used to generate different dance movements, which were perceived 
by human observers as conveying distinct emotions in a user study [12]. In [13], the proposed quantification by Nakata et al. was adapted for extracting characteristic full-body human movements. An agreement between the quantification and an expert annotation was reported only for detecting the extremes of Time and Weight, without providing an explicit comparison in terms of statistical correlation. Furthermore, the quantified Shape components were not verified in the study. In [14], quantified Laban Body, Effort (Weight, Time, Space, and Flow), and Shape Flow components were used for full-body motion indexing and retrieval. The quantified components were not verified in comparison to an expert annotation. Camurri et al. [15] and Glowinski et al. [16] propose quantitative features (motion fluency, impulsiveness, energy computed as the weighted sum of the limbs' kinetic energy, spatial extent) inspired by Laban Effort components for automatic recognition of video-tracked affective movements. The application of Laban Effort and Shape is also reported for movement generation, in which movement physical features (e.g., position) are derived using a set of specified Laban Effort and Shape components in [17] and features inspired by social psychology reports on bodily expression of affect in [18]. In contrast to [17] and [18], who use specified Laban levels to generate movements, our work aims at quantifying the Laban components from observed human movements using measurable physical movement features.

In this work, the Laban Effort quantifications from [13], [14] are adapted for hand and arm movements. We propose a continuous measure of Laban Effort components, unlike [13] and [14], which quantify the components as boolean features. In addition, a quantification for Shape Directional of hand and arm movements is proposed. The quantified Laban components are verified by assessing their statistical correlation with annotations provided by a certified movement analyst (CMA) for a set of affective hand and arm movements. It is shown that the proposed quantifications provide reliable measures of Laban components, which are in congruence with CMA annotations.

\section{LABAN EFFORT AND SHAPE}

Laban has four major components: Body $^{2}$, Space, Shape, and Effort. Body indicates the active body parts, and the sequence of their involvement in the movement. Space defines where in space the movement is happening, and directions of the body and body parts. Shape characterizes the bodily form, and its changes in space. Effort describes the inner attitude toward the use of energy. In other words, Body and Space describe What one does through a movement, whereas Effort and Shape describe How the movement is performed [9].

Bartenieff presented Effort and Shape as a complete system for the objective study of different types of movements, from behavioural and expressive perspectives [9]. Bartenieff emphasizes that emotions and their intensity can be precisely observed through the Effort components. Therefore, Effort and Shape are the most relevant for the study of affective movements. In particular, when the motion trajectory is known, Effort and Shape can be used to encode qualitative modulations of the specified motion trajectory for conveying desired affective expressions. Effort and Shape represent affective movements in terms of four components of Effort (Table I) and three components of Shape (Table II).

\footnotetext{
${ }^{2}$ In this paper, Laban terms are capitalized.
}

TABLE I. LABAN EFFORT COMPONENTS ADAPTED FROM [9], [19]

\begin{tabular}{lcl}
\hline \hline & Extremes & Example \\
\hline Space: Attention to & Direct & Pointing to a particular spot \\
surroundings & Indirect & Waving away bugs \\
Weight: Sense of the & Light & Dabbing paint on a canvas \\
impact of ones movement & Strong & Punching \\
Time: Sense of & Sustained & Stroking a pet \\
urgency & Sudden & Swatting a fly \\
Flow: Attitude toward & Free & Waving wildly \\
bodily tension and control & Bound & Carefully carrying a cup of hot liquid \\
\hline
\end{tabular}

III. LABAN EFFORT AND SHAPE QUANTIFICATIONS

In this work, two Laban Effort quantifications for fullbody movements (Weight, Time, Space quantifications from [13], and Weight, Time, Space, Flow quantifications from [14]) are adapted for hand and arm movements. Furthermore, an approach to quantify Shape Directional is proposed. The adapted quantifications from [13] and [14] are referred to as Q1 and Q2, hereafter. It should be emphasized that in both [13] and [14], Laban Effort components are considered as boolean features (high/low feature), and their quantification as a boolean feature depends on the definition of a suitable threshold. Furthermore, the quantifications for Time, Weight, Space, and Flow Effort in [14] store the time frames at which a Sudden, Strong, Direct, and Bound motion starts and ends. In this work, the Effort components are quantified as continuous quantities. The efficacy of the quantification approaches will be compared with each other, and with CMA annotations of the subject movements. Note that [13] applies the quantification to the aggregate set of body parts involved in a movement, while [14] quantifies Effort components for individual body parts.

Weight Effort: Inspired by [13], the maximum of the sum of the kinetic energy of the moving body parts is used to estimate Weight Effort. The higher the maximum of the kinetic energy, the Stronger the Weight. The sum of the kinetic energy for the hand and arm at time $t_{i}$ is:

$$
\begin{aligned}
& E\left(t_{i}\right)=E^{\text {Hand }}\left(t_{i}\right)+E^{A r m}\left(t_{i}\right), \text { where } \\
& E^{\text {Hand }}\left(t_{i}\right)=\sum_{j=\text { Fingers }} E^{j}\left(t_{i}\right) \\
& E^{\text {Arm }}\left(t_{i}\right)=E^{\text {UpperArm }}\left(t_{i}\right)+E^{\text {Forearm }}\left(t_{i}\right) .
\end{aligned}
$$

For instance, the kinetic energy of the forearm at time $t_{i}$ is computed as:

$$
E^{\text {Forearm }}\left(t_{i}\right)=\alpha_{\text {Forearm }} v^{\text {Forearm }}\left(t_{i}\right)^{2},
$$

where $\alpha_{\text {Foregrm }}$ is the mass coefficient for the forearm and $v^{\text {Forearm }}\left(t_{i}\right)^{2}$ is the speed of the forearm at time $t_{i}$. The proposed Weight Effort for a movement of length $T$ is then determined as:

$$
\text { Weight }_{Q 1}=\max \left(E\left(t_{i}\right)\right) \forall i \in[0, T] .
$$

In [12], the mass coefficients for different body parts of a dancing pet robot were set based on their "visual mass and conspicuousness" in the range of 1 to 4 (e.g., $\alpha_{\text {trunk }}=4$ and $\alpha_{\text {arm }}=2$ ). In [13], the mass coefficients for different human body parts were set to 1 . In the present work, mass coefficients are set to 1 for all the body parts.

In [14], Weight is quantified using deceleration of motion: Strong (large deceleration) or Light (little or no deceleration). In the present work this is adapted such that for each hand and arm movement, the maximum of the deceleration time-series for the $k^{t h}$ body part is used as the Weight component for that body part $\left(\right.$ Weight $\left.{ }_{Q 2}^{k}\right)$. 
TABLE II. LABAN ShaPE COMPONENTS [20].

\begin{tabular}{lll}
\hline \hline & Elements & Example \\
\hline $\begin{array}{l}\text { Shape Flow: is self-referential and defines readjustments of the } \\
\text { whole body for internal physical comfort*. }\end{array}$ & Growing & Self-to-self communication, stretching to yawn \\
$\begin{array}{l}\text { Directional: is goal-oriented and defines the pathway to connect } \\
\text { or bridge to a person, object, or location in space. }\end{array}$ & Exhaling with a sigh \\
$\begin{array}{l}\text { Shaping/Carving: is process-oriented and is the three dimen- } \\
\text { sional "sculpting" of body oriented to creating or experiencing } \\
\text { volume in interaction with the environment. }\end{array}$ & Swinging the arm forward to shake hands & Spoke-like \\
\end{tabular}

*Shape Flow remains as a support for Directional and Shaping/Carving movements [21].

Time Effort: Based on the proposed quantification in [13], the weighted sum of the accelerations of the moving body parts is used to estimate the Time Effort for hand and arm movements. The weights are the mass coefficients similar to the $W$ eight $t_{Q 1}$. The acceleration ${ }^{3}$ for the $k^{\text {th }}$ body part at time $t_{i}$ is:

$$
a^{k}\left(t_{i}\right)=v^{k}\left(t_{i}\right)-v^{k}\left(t_{i-1}\right) .
$$

Sudden movements are characterized by larger values in the weighted acceleration time-series as compared to Sustained movements. In particular, in the present study, the maximum of the computed weighted acceleration time-series is used to describe Time Effort of hand and arm movements $\left(\right.$ Time $_{Q 1}$ ).

Using [14], the Time Effort for a movement of length $T$ is determined as the net acceleration accumulated at the body parts over time (Equation 5). In Equation 5, $\mathbf{v}^{k}\left(t_{i}\right)$ represents the Cartesian velocity of the $k^{\text {th }}$ body part at time $t_{i}$.

$$
\operatorname{Time}_{Q 2}^{k}=\sum_{i=2}^{T} \frac{\left.\mid \mathbf{v}^{k}\left(t_{i}\right)-\mathbf{v}^{k}\left(t_{i-1}\right)\right) \mid}{t_{i}-t_{i-1}} .
$$

Space Effort: The Space quantification from [13] is adapted to measure the Directness of hand and arm movements using the inner product of tangents of torso and wrist trajectories at every time instance. If a movement is Direct, then the resulting inner product time-series would be strictly monotonic or constant, whereas an Indirect movement would demonstrate a fluctuating time-series with multiple peaks (or changes in direction). Therefore, to quantify Space Effort, the number of peaks in the resulting Space time-series is counted, and the Space Effort component is determined on a scale of 1 to 5 through a binning process.

$$
\text { Space }_{Q 1}=\# \text { of peaks }\left(\mathbf{v}^{W}\left(t_{i}\right) \cdot \mathbf{v}^{T}\left(t_{i}\right) \forall i \in[0, T]\right),
$$

where $\mathbf{v}^{W}\left(t_{i}\right)$ and $\mathbf{v}^{T}\left(t_{i}\right)$ represent tangents to wrist and torso trajectories at time $t_{i}$, respectively.

Using the Space quantification from [14], the Space Effort at an upper body part is quantified as the ratio of the displacement to the net distance travelled by the body part. In the present study, Space $Q_{Q 2}^{k}$ represents the Space component for the $k^{\text {th }}$ body part and is computed for a movement of length $T$ as:

$$
\operatorname{Space}_{Q 2}^{k}=\frac{\sum_{i=2}^{T}\left|\mathbf{P}^{k}\left(t_{i}\right)-\mathbf{P}^{k}\left(t_{i-1}\right)\right|}{\left|\mathbf{P}^{k}(T)-\mathbf{P}^{k}\left(t_{1}\right)\right|},
$$

where $\mathbf{P}^{k}\left(t_{i}\right)$ is the Cartesian position of the $k^{t h}$ body part at time $t_{i}$.

\footnotetext{
${ }^{3}$ In the quantifications, the derivatives of motion trajectories, used to compute velocity, acceleration, and jerk, are low-pass filtered to remove the high-frequency noise.
}

Flow Effort for a body part $k$ is computed as the aggregated jerk over time as proposed in [14] (Equation 8). Jerk is the third order derivative of the position.

$$
\text { Flow }_{Q 2}^{k}=\sum_{i=2}^{T} \frac{\left|\mathbf{a}^{k}\left(t_{i}\right)-\mathbf{a}^{k}\left(t_{i-1}\right)\right|}{t_{i}-t_{i-1}},
$$

where $\mathbf{a}^{k}\left(t_{i}\right)$ is the Cartesian acceleration of the $k^{t h}$ body part at time $t_{i}$.

Shape Directional defines the pathway along which a movement is executed. Shape Directional can be 'Spoke-like' or 'Arc-like'. Spoke-like Directional movements travel on a straight line from the body center or return on a straight line to the body center [21]. The pathway of Arc-like movements is characterized by a flat arc starting at the side and moving forward and upward [21]. Furthermore, Arc-like Directional movements can have a flat arc in the horizontal plane while travelling forward and sideways(and likewise in the reverse direction).

To capture Shape Directional, which wasn't considered in either of [14] or [13], we propose to use the average curvature of the movement in a 2 dimensional (2D) plane within which the largest displacement occurs. To capture the 2D plane with maximum displacement, we apply multi-variate principal component analysis (PCA) [22] on the 3D Cartesian trajectories of the upper body joints and extract the top two dimensions. The extracted dimensions span the 2D plane where the maximum displacement occurs. Next, the average 2D curvature within the PCA-extracted 2D plane (xy plane) for a movement of length $T$ is computed as follows:

$$
\kappa=\frac{1}{T} \sum_{i=1}^{T} \frac{\sqrt{\left(\ddot{y}\left(t_{i}\right) \dot{x}\left(t_{i}\right)-\ddot{x}\left(t_{i}\right) \dot{y}\left(t_{i}\right)\right)^{2}}}{\left(\dot{x}^{2}\left(t_{i}\right)+\dot{y}\left(t_{i}\right)^{2}\right)^{3 / 2}},
$$

where $\dot{x}\left(t_{i}\right)$ and $\ddot{x}\left(t_{i}\right)$ indicate the first and second derivatives of $x$ trajectory at time $t_{i}$, respectively.

\section{A. Affective hand and arm dataset}

\section{EXPERIMENTS}

On the one hand, an affective expression can be communicated through a number of kinematically different movements, and on the other hand, there exist kinematically similar movements that convey distinct affective expressions. Therefore, for exploratory analysis, a movement dataset is needed that provides exemplars representing these nuances in affective expressions. In this work, six hand and arm motion paths were designed in consultation with a CMA, and used by a professional actor to convey six basic Ekman emotions (i.e., anger, happiness, sadness, fear, disgust, surprise [23]) one at a time. Therefore, each demonstrated movement, in its entirety, was intended to convey a specific emotion. Table III describes 
TABLE III. HAND AND ARM MOTION PATHS*

\begin{tabular}{cl}
\hline \hline Motion path & Description \\
\hline A & $\begin{array}{l}\text { From Self, right arm down along side to forward mid Level, } \\
\text { reaching to take something, palm up palm remains open. }\end{array}$ \\
B & $\begin{array}{l}\text { Similar motion as A, but in the reverse direction. } \\
\text { C }\end{array}$ \\
& $\begin{array}{l}\text { Right palm open on upper chest, opening and extending right } \\
\text { arm fully directly in front of the right shoulder at forward mid- } \\
\text { level, with the palm facing left, perpendicular to the floor similar } \\
\text { to the hand-shake position. }\end{array}$ \\
D & $\begin{array}{l}\text { Similar motion as C, but in the reverse direction. } \\
\text { Eight arm is extended forward at mid-level with open palm } \\
\text { facing down and the hand parallel to the floor. Moving backward } \\
\text { ending with the open palm facing forward near the right } \\
\text { shoulder. } \\
\text { Similar motion as E, but in the reverse direction. }\end{array}$ \\
F & During these movements, the wrist and finger orientations remain constant.
\end{tabular}

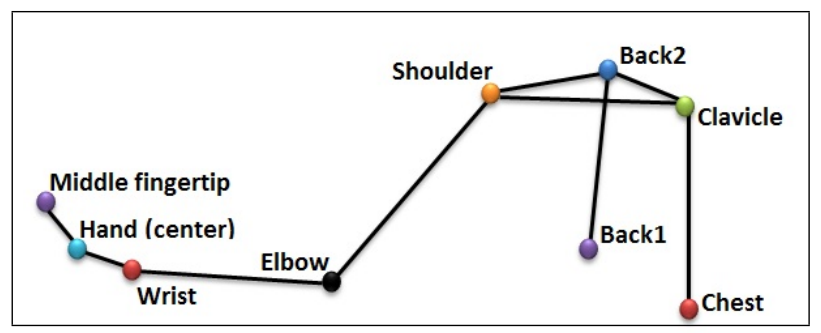

Fig. 1. Upper body model used in Laban quantification.

the designed hand and arm motion paths. For each pair of motion path and emotion, 5 trials were recorded.

The study received ethics approval from the Office of Research Ethics, University of Waterloo. The motion capture was done using the MotionAnalysis ${ }^{4}$ optical motion capture system with a sampling frequency of $200 \mathrm{~Hz}$, at the University of Waterloo and in the presence of the CMA and one of the researchers. Informed consent was obtained from the actor prior to the motion capture session. The marker set used for the motion capture is shown in Figure 1.

\section{B. CMA annotation}

Laban movement annotations provided by the CMA are used to investigate the efficacy of the proposed Laban quantifications for the hand and arm movements by computing the statistical correlation between the CMA-annotated and quantified Laban components. A high correlation between the CMA-annotated and quantified Laban components verifies the efficacy of the quantification.

For this purpose, an annotation questionnaire was designed in consultation with the CMA in which Weight, Time, Space, and Flow Effort of a movement were rated by the CMA on a 5point Likert scale, while watching the video of the movement. For each Effort component, two extra 5-point Likert scales were also provided to allow for annotating subintervals along the movement with distinct Effort qualities (if any). The 5point Likert scale spans between poles of Effort components. Furthermore, an open-ended text box was provided for each Effort component to accommodate additional comments that the CMA might have on a particular movement and annotation. In the videos, the actor's face was masked and the videos were muted.

The questionnaire also included annotation of Shape Directional (options: Arc-like/Spoke-like), Shaping/Carving (options: yes/not significant). Shape Flow is self-referential and defines readjustments of the whole body for internal physical

\footnotetext{
${ }^{4}$ Motion Analysis Corporation, www.motionanalysis.com
}

comfort [20]. Therefore, Shape Flow can not be observed in the isolated and prescribed hand and arm movements used in this study, and it was excluded from the annotation and quantification analysis. Furthermore, a visual observation of the hand and arm movements revealed to the CMA that Shaping/Carving occurs only slightly in the fingers for some of the movements, which is probably due to the prescribed motion paths and no-movement constraints placed on the wrist and fingers. Therefore, the Shaping/Carving is not significant for the hand and arm movements with the kinematic model used in this work (Figure 1), and it was also excluded from quantification analysis.

A total of 44 movements were annotated, which include the following motion-emotion pairs: 3 F-anger, 3 F-sadness, 3 F-disgust, 3 F-fear, 2 E-surprise, 2 E-fear, 1 E-disgust, 1 E-sadness, 1 E-happiness, 1 E-anger, 2 D-anger, 1 Dsadness, 2 D-fear, 2 C-anger, 2 C-happiness, 2 C-sadness, 2 C-disgust, $2 \mathrm{C}$-fear, $2 \mathrm{C}$-surprise, $1 \mathrm{~B}$-anger, $1 \mathrm{~B}$-happiness, 1 B-sadness, 1 B-disgust, 1 B-fear, 1 B-surprise, 1 A-happiness. We assume that each movement in the dataset has unique Time, Weight, Space, and Flow Effort qualities and therefore, we only consider the CMA-annotated movements with single Effort annotations (sample size column in Table IV). This assumption is important as we are after movement cues most salient to different Effort and Shape components that can uniquely represent varying levels of these components.

\section{RESUlTS AND Discussion}

The correlations between the quantified and CMAannotated Effort and Shape components are computed based on the Pearson linear correlation coefficient. Note that the proposed quantification measures are continuous and not discrete classes/labels, therefore classification accuracy measures such as precision and recall are not reported. Furthermore, the significance of the obtained correlations is evaluated at $p<0.05$ with a null hypothesis $H^{0}$ stating: there is no correlation, $r=0$, between the CMA-annotated and quantified Laban component $x$, where $x=\{$ Space, Time, Weight, Flow, Shape Directional $\}$. $p$-values represent the probability of getting a correlation as large as the observed correlation by random chance, when the true correlation is zero. Table IV shows the resulting correlations between the quantified and CMAannotated Effort components and their significance. The Q1 Effort quantifications are obtained for aggregate set of body parts involved in the movements, whereas the Q2 Effort quantifications are obtained for each body part, separately.

There are high and significant correlations between the CMA-annotated and quantified Laban components for Weight and Time Effort, whereas there are lower correlations between the quantified and CMA-annotated Space and Flow Effort. Two approaches were used to measure Weight Effort: 1) the maximum of the kinetic energy of the upper body parts $\left(\right.$ Weight $\left._{Q 1}\right)$, and 2) the maximum of the deceleration at different upper body parts $\left(W\right.$ eight $\left._{Q 2}\right)$. Considering a unit distance travelled by a body part, the deceleration of that body part is proportional to the rate of change in its kinetic energy. The maximum of the deceleration implies the maximum absorption of the kinetic energy [14] e.g., at the end of a punching movement. Therefore, the peak of the deceleration and the peak of the kinetic energy (the difference between the maximum and minimum of the kinetic energy as the minimum of the kinetic energy is zero) are both measuring the maximum 

INDICATES A SIGNIFICANT CORRELATION.

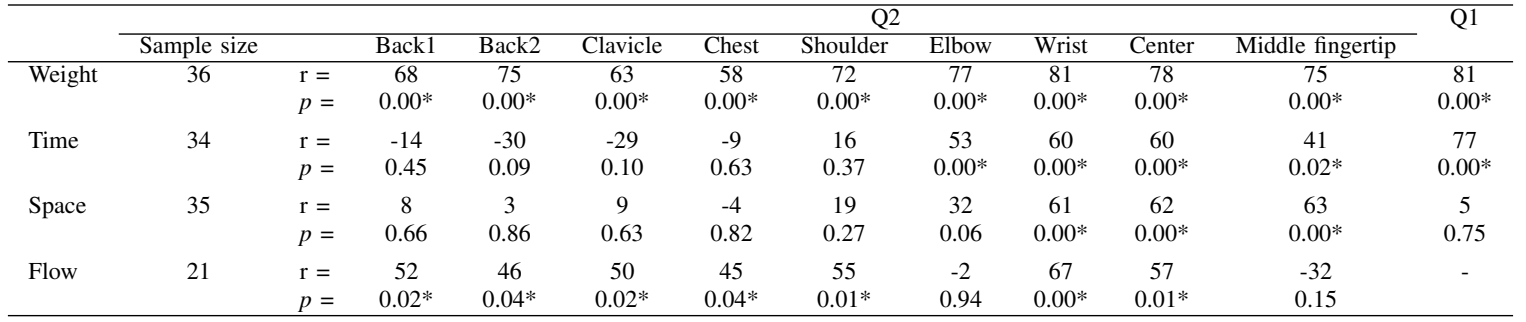

absorption of the kinetic energy. However, the peak of the kinetic energy of the body parts $\left(\right.$ Weight $\left._{Q 1}\right)$ provides a more accurate measure for Weight Effort $(r=81 \%)$ as it considers the maximum discharge of the kinetic energy of all the body parts.

Bartenieff described Sudden Time as react quickly or immediately and Sustained Time as take time, moving slowly and lingering in time [9]. Considering these descriptions, Sudden movements should demonstrate larger accelerations, whereas Sustained movements are characterized with lower or no acceleration. In our study, the peak acceleration of the upper body parts $\left(\right.$ Time $\left._{Q 1}\right)$ results in a higher correlation with the CMA-annotated Time $(r=77 \%)$ as compared to the aggregated acceleration $\left(\right.$ Time $_{Q 2}: r=60 \%$ at wrist). It is possible that the aggregated acceleration produces a large quantity for Sustained movements, which are generally characterized as lingering in time (resulting in a longer duration as compared to Sudden movements), and as a result identifies them as Sudden movements. Normalizing the aggregated acceleration by the length of the movements results in an improved correlation $(r$ $=71 \%$ at wrist) as compared to the unnormalized aggregated acceleration used in [14]. However, this is still lower than the correlation for the maximum acceleration measure. The normalized aggregated acceleration is not an appropriate measure for Time Effort as it represents the average acceleration and fails to capture abrupt changes in the velocity. Therefore, the maximum acceleration at the upper body parts $\left(\right.$ Time $\left._{Q 1}\right)$ is a more appropriate measure for Time Effort.

The kinematic cues (velocity and acceleration) used for quantification of Weight and Time Effort are similar to those reported as relevant cues to these components in the movement literature [24], [25], [26], [27], [15], [16]. The average quantified Weight $\left(\right.$ Weight $\left._{Q 1}\right)$ and Time $\left(\right.$ Time $\left._{Q 1}\right)$ Effort for the affective hand and arm movements are shown in Figure 2. The sad and angry movements are clearly distinguishable from the rest of the movements using the quantified Time and Weight. The angry movements are characterized by Strong Weight and Sudden Time, whereas the sad movements are characterized by Light Weight and Sustained Time. The average Weight for the remaining emotions is Light, however none of them exceeds the Lightness of the sad movements. The Time for the surprised movements is slightly oriented toward Sudden Time, whereas the Time for the fearful, disgusted, and happy movements is slightly Sustained. These results can be used to study the capability of the isolated hand and arm movements to convey distinct basic emotions.

The majority of the movements (31/35) are annotated by the CMA as Direct Space, only 2 are annotated as Indirect and the remaining 2 are annotated as Spaceless (no attention to Space). Therefore, due to the imbalance in the sample size
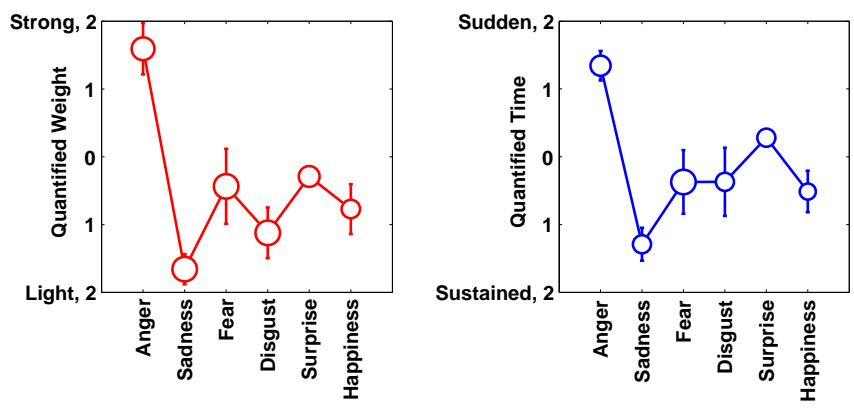

Fig. 2. Average (and standard error) quantified Weight and Time for the affective hand and arm movements. y-axis labels are assigned to correspond to the 5-point Likert scale used in the annotation questionnaire. The circles and their sizes indicate the average value and sample size, respectively.

(lack of adequate samples at different levels of Space), it is difficult to validate the proposed Space quantifications for hand and arm movements. Furthermore, Space Effort describes the actor's focus (single-focused vs multi-focused) and other visual cues (facial movements) might be needed to better evaluate Space. For instance, an expansive arm movement can be used to greet several arriving parties (multi-focused, Indirect) or a single arriving person (single-focused, Direct), which would be difficult to annotate without additional contextual information.

The spatial stopping constraint in the motion paths (Table III) contributes to having movements with multiple Flow qualities, as it turns a movement to Bound Flow toward the end even if it begins as a Free Flow movement. This resulted in Flow being the Effort component with the most multiple annotations for each movement (23 out of 44 movements). Using the magnitude of jerk, derivative of acceleration, for quantifying the Flow $\left(\right.$ Flow $\left._{Q 2}\right)$, a significant correlation as high as $67 \%$ is obtained at the wrist. Other studies also relate Flow to the jerkiness of the motion trajectory (e.g., [26], [27], [15]).

Using the average curvature of the movement projections in the PCA-extracted 2D planes, a correlation of $81 \%(p<$ 0.0001 ) between the quantified and CMA-annotated Shape Directional is obtained. Figure 3 shows the quantified values for Shape Directional, in which Arc-like Shape Directional (as annotated by the CMA) and a suitable threshold for labelling are shown as red circles and a dashed line, respectively. Applying the threshold, the movements are divided into two bins of Spoke-like and Arc-like, and a Phi correlation ${ }^{5}$ of $93 \%$ is obtained between the binned-quantified and CMA-annotated Shape Directional. The high correlation results for Shape

\footnotetext{
${ }^{5}$ Pearson correlation computed between two binary variables returns Phi correlation.
} 


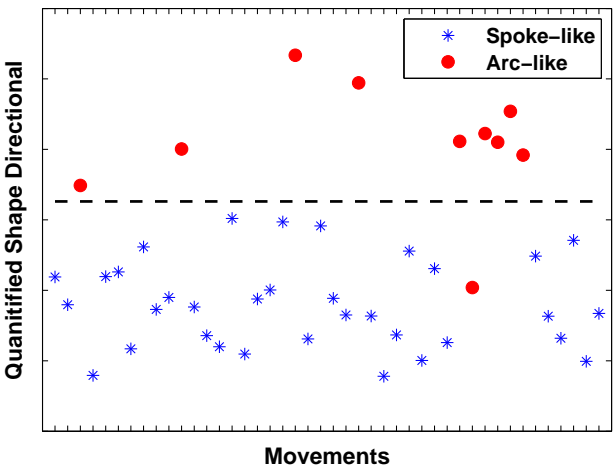

Fig. 3. Quantified Shape Directional for different movements. The dashed line illustrates a suitable threshold value.

Directional verify the efficacy of the proposed measure for identifying the Arc-like or Spoke-like Shape of the movements.

\section{CONCLUSIONS}

The Laban Effort and Shape provide a systematic approach to describe expressive qualities of a movement, which can benefit the computational analysis of affective movements. To enable the application of the Laban Effort and Shape components in computational analysis, these components need to be quantified. In this study, two approaches for quantifying the Laban Effort are adapted for hand and arm movements. Furthermore, an approach for quantifying Shape Directional of hand and arm movements is proposed. The quantifications are based on measurable physical movement cues. The efficacy of the quantifications is evaluated in comparison to the annotations provided by a CMA for an affective hand and arm movement dataset. The dataset consists of different affective expressions communicated through a set of predefined hand and arm movements by a professional actor. The proposed Laban Effort and Shape quantifications are used to characterize the subtle modulations in the execution of the movements to convey a desired affective expression.

The results show that the quantified and annotated components are highly correlated, specifically for the Time and Weight Effort, and Shape Directional. All the high correlations reported in this study are found to be statistically significant. Due to the imbalance in the dataset (majority Direct movements), the Space quantification can not be verified and needs a larger sample size at different levels of Space Effort to be validated. Furthermore, the stopping constraint on the designed motion paths, to some extent, limits the movement variations along Flow Effort. However, the proposed Flow quantification can fairly predict CMA's Flow annotation (67\% correlation). In future works, hand and arm movements with varied Flow qualities will be considered to further verify the proposed measure.

A mapping between affective categories/dimensions and different levels of the quantified Laban Effort and Shape components will be explored in future work to identify Laban components and their magnitudes salient to different affective categories and dimensions.

\section{REFERENCES}

[1] C. Breazeal, Designing sociable robots. The MIT Press, 2004.

[2] P. Beesley, "Hylozoic soil," Leonardo, vol. 42, no. 4, pp. 360-361, 2009
[3] S. J. Park, G. Chae, C. MacDonald, R. Stein, S. Wiedenbeck, and J. Kim, "Amara: The affective museum of art resource agent," in $\mathrm{CHI}$ '12 Extended Abstracts on Human Factors in Computing Systems, ser. CHI EA '12. ACM, 2012, pp. 1035-1038.

[4] J. Burgoon, D. Buller, and W. Woodall, Nonverbal communication. Allyn \& Bacon, 2010.

[5] R. Birdwhistell, Kinesics and context: Essays on body motion communication. Univ of Pennsylvania Pr, 1970.

[6] P. Ekman and W. Friesen, "The repertoire of nonverbal behavior: Categories, origins, usage, and coding," SEMIOTICA, vol. 1, no. 1, pp. 49-98, 1969.

[7] R. Laban and F. Lawrence, Effort. Macdonald and Evans, 1947.

[8] R. Laban and L. Ullmann, The mastery of movement. ERIC, 1971.

[9] I. Bartenieff, Effort-Shape analysis of movement: The unity of expression and function. Albert Einstein College of Medicine, Yeshiva University, 1965

[10] A. Samadani, A. Ghodsi, and D. Kulić, "Discriminative functional analysis of human movements," Pattern Recognition Letters, 2013. [Online]. Available: http://www.sciencedirect.com/science/article/pii/S0167865512004242

[11] G. Castellano, S. Villalba, and A. Camurri, "Recognising human emotions from body movement and gesture dynamics," in Affective Computing and Intelligent Interaction, ser. Lecture Notes in Computer Science, A. Paiva, R. Prada, and R. Picard, Eds. Springer Berlin Heidelberg, 2007, vol. 4738, pp. 71-82.

[12] T. Nakata, T. Mori, and T. Sato, "Analysis of impression of robot bodily expression," Journal of Robotics and Mechatronics, vol. 14, no. 1, pp. $27-36,2002$

[13] K. Hachimura, K. Takashina, and M. Yoshimura, "Analysis and evaluation of dancing movement based on LMA," in IEEE International Workshop on Robot and Human Interactive Communication, ROMAN. IEEE, 2005, pp. 294-299.

[14] M. Kapadia, I. Chiang, T. Thomas, N. I. Badler, and J. Kider, "Efficient Motion Retrieval in Large Databases," in Proceedings of the symposium on Interactive 3D graphics and games, ser. I3D. ACM, 2013.

[15] A. Camurri, I. Lagerlöf, and G. Volpe, "Recognizing emotion from dance movement: comparison of spectator recognition and automated techniques," International Journal of Human-Computer Studies, vol. 59, no. 1, pp. 213-225, 2003.

[16] D. Glowinski, N. Dael, A. Camurri, G. Volpe, M. Mortillaro, and K. Scherer, "Toward a minimal representation of affective gestures," Affective Computing, IEEE Transactions on, vol. 2, no. 2, pp. 106-118, 2011.

[17] D. Chi, M. Costa, L. Zhao, and N. Badler, "The EMOTE model for effort and shape," in Proceedings of the 27th annual conference on Computer graphics and interactive techniques, ser. SIGGRAPH '00, 2000, pp. 173-182.

[18] B. Hartmann, M. Mancini, and C. Pelachaud, "Towards affective agent action: Modelling expressive eca gestures," in Intelligent User Interfaces 2005 Workshop on Affective Interaction, 2005.

[19] D. M. Chi, "A motion control scheme for animating expressive arm movements," Ph.D. dissertation, University of Pennsylvania, 1999.

[20] P. Hackney, Making connections: Total body integration through Bartenieff fundamentals. Routledge, 2004.

[21] T. Casciero, Laban Movement Studies and Actor Training: An Experiential and Theoretical Course for Training Actors in Physical Awareness and Expressivity. UMI Dissertation Services, 1998.

[22] I. Jolliffe and MyiLibrary, Principal component analysis. Wiley Online Library, 2002, vol. 2.

[23] P. Ekman, "Are there basic emotions?" Psychological Review, vol. 99, no. 3, pp. 550-553, 1992

[24] M. Gross, E. Crane, and B. Fredrickson, "Methodology for assessing bodily expression of emotion," Journal of Nonverbal Behavior, vol. 34, pp. 223-248, 2010.

[25] T. Lourens, R. Van Berkel, and E. Barakova, "Communicating emotions and mental states to robots in a real time parallel framework using Laban movement analysis," ROBOT AUTON SYST, vol. 58, no. 12, pp. 1256-1265, 2010.

[26] E. Barakova and T. Lourens, "Expressing and interpreting emotional movements in social games with robots," Pers Ubiquit Comput, vol. 14, pp. 457-467, 2010.

[27] D. Petersen, "Space, time, weight, and flow: suggestions for enhancing assessment of creative movement," Physical Education and Sport Pedagogy, vol. 13, no. 2, pp. 191-198, 2008. 